\title{
Determination of Creatinine in Human Urine with Flow Injection Tandem Mass Spectrometry
}

\author{
Mareile Niesser Berthold Koletzko Wolfgang Peissner \\ Division of Metabolic and Nutritional Medicine, Dr. von Hauner Children's Hospital, University of Munich \\ Medical Centre, Munich, Germany
}

\section{Key Words}

Creatinine $\cdot$ Human urine $\cdot$ Tandem mass spectrometry $\cdot$

Flow injection analysis $\cdot$ Qualifier ion monitoring

\begin{abstract}
Background/Aims: Excretion of urinary compounds in spot urine is often estimated relative to creatinine. For the growing number of liquid chromatography-tandem mass spectrometry (LC-MS/MS) assays of urine-excreted molecules, a fast and accurate method for determination of creatinine is needed. Methods: A high-throughput flow injection tandem mass spectrometry method for exact quantitation of creatinine in urine has been developed and validated. Sample preparation used only two-step dilution for protein precipitation and matrix dilution. Flow injection analysis without chromatographic separation allowed for total run times of 1 min per sample. Creatinine concentrations were quantitated using stable isotope dilution tandem mass spectrometry. Selectivity and coelution-free quantitation were assured by qualifier ion monitoring. Results: Method validation revealed excellent injection repeatability of $1.0 \%$ coefficient of variation (CV), intraday precision of $1.2 \% \mathrm{CV}$ and interday precision of $2.4 \% \mathrm{CV}$. Accuracy determined from standard addition experiments was $106.1 \pm 3.8 \%$. The linear calibration range was adapted to physiological creatinine concentrations. Comparison of quantitation results
\end{abstract}

with a routinely used method (Jaffé colorimetric assay) proved high agreement $\left(R^{2}=0.9102\right)$. Conclusions: The new method is a valuable addition to the toolbox of LC-MS/MS laboratories where excretion of urinary compounds is studied. The 'dilute and shoot' approach to isotope dilution tandem mass spectrometry makes the new method highly accurate as well as cost- and time-efficient.

Copyright $\odot 2012$ S. Karger AG, Basel

\section{Introduction}

Liquid chromatography-tandem mass spectrometry (LC-MS/MS) is gaining importance as a convenient and flexible tool for quantitative determination of a wide range of drugs, toxins and endogenous molecules from urinary samples. To account for variations of urine dilution in spot urine samples, calculation of the creatinine ratio is often performed $[1,2]$. The method presented here is intended to be useful when additional determination of urinary creatinine is desirable along with LC-MS/MS assays of substances excreted in urine, e.g. to report drug or drug metabolite concentrations in urine [3], to evaluate specific markers of oxidative stress in biological studies [4] or to detect lung cancer risk from exposure to cigarette smoke [5].

\section{KARGER \\ Fax +4161306 1234 \\ E-Mail karger@karger.ch}

www.karger.com
(C) 2012 S. Karger AG, Basel

$0250-6807 / 12 / 0614-0314 \$ 38.00 / 0$

Accessible online at:

www.karger.com/anm
Berthold Koletzko, MD, Professor of Pediatrics

Dr. von Hauner Children's Hospital, University of Munich Medical Centre Lindwurmstrasse 4

DE-80337 Munich (Germany)

E-Mail office.koletzko@med.uni-muenchen.de 
Creatinine is a break-down product of creatine phosphate, produced primarily in muscle, released into blood and excreted in urine $[6,7]$. Creatinine is released from muscle into the blood stream at a highly constant rate which is proportional to the muscle mass of the individual [7-9]. The kidneys readily excrete creatinine by glomerular filtration and proximal tubular secretion without relevant tubular reabsorption $[9,10]$. To account for highly variable dilution of spot urine samples, concentrations of urinary excreted compounds are often reported after normalization to creatinine concentration [3-5].

Colorimetric determinations of the picrate salt or assays based on enzymatic reactions are commonly used in clinical chemistry [11-13]. Creatinine assays examining the reaction of creatinine and picric acid were first reported by Jaffé [14]. To address well-known selectivity problems of the Jaffé method, many modifications have been proposed, but none of them has eliminated all interferences $[15,16]$. Various endogenous and exogenous substances reacting with alkaline picrate are partly contributing to measured creatinine concentration as 'noncreatinine chromogens' $[10,15]$.

Additional methods, applying extensive matrix preseparation, have been proposed for the determination of creatinine in urine, for instance capillary electrophoresis [9, 17], gas chromatography [7], liquid chromatography combined with UV [18] and recently liquid chromatography coupled to mass spectrometry $[5,19,20]$.

Flow injection tandem mass spectrometry (FIA-MS/ MS) was introduced more than 2 decades ago for detection of metabolite profiles indicative of inherited metabolic disease from dried blood spots [21, 22]. Similar methods are now used in many nationwide neonatal screening programs $[23,24]$. In the fields of pesticide determination [25-27], therapeutic drug monitoring [28] and analysis of adulterated dietary supplements [29], FIA-MS/MS has recently been presented as an adequate alternative to LC-MS/MS. For FIA-MS/MS the mass spectrometer is directly coupled to a pump delivering a continuous flow of eluent solvents, whereas the LC column compartment is bypassed. Injection of samples into the solvent flow (usually using an autosampler device) results in well-formed injection peaks. FIA-MS/MS in complex biological matrices like plasma or urine is typically done after straightforward sample dilution in organic solvents for precipitation of proteins and reduction of ion suppression effects, an approach known as 'dilute and shoot'. The two most important analytical challenges of FIA-MS/MS are: (1) assessment and exclusion of coelution bias, which is here addressed by qualifier ion monitoring $[29,30]$, and (2) correct quantification in the presence of significant ion suppression, which is best achieved by using properly matched stable isotope-labeled internal standards (SIL-IS) [31]. If properly applied, FIA-MS/MS provides high precision, short run times and remarkable cost savings due to elimination of time-consuming and possibly error-prone procedures of sample cleanup and pre-separation [27].

The aim of our study was to develop a rapid and costeffective FIA-MS/MS method for the determination of creatinine in human urine. The method was used in combination with LC-MS/MS determination of endogenous urinary metabolites in a large-scale clinical intervention trial [Niesser et al., unpublished]. The highly precise and time-efficient FIA-MS/MS quantitation of creatinine from the same urine sample enabled estimation of metabolite excretion from its ratio to creatinine.

\section{Material and Methods}

\section{Chemicals, Reagents and Reference Standards}

Creatinine was purchased from Sigma-Aldrich Chemistry (Taufkirchen, Germany) and $\mathrm{D}_{3}$-creatinine (creatinine-N-methyl- $D_{3}$ ), which was used as internal standard (IS), from CDN Isotopes (Dr. Ehrenstorfer GmbH, Augsburg, Germany). HPLCgrade water and formic acid were purchased from Sigma-Aldrich Chemistry. Formic acid in acetonitrile (0.1\%, LC-MS grade) was ordered from J.T. Baker (Avantor Performace Materials B.V., Deventer, The Netherlands). Synthetic urine was supplied by Cerilliant Corporation (Round Rock, Tex., USA).

Creatinine and $\mathrm{D}_{3}$-creatinine stock solutions were prepared in a mixture of acetonitrile and water (50:50), and stored at $-20^{\circ} \mathrm{C}$.

\section{Sample Preparation}

Spot and 24-hour urine samples from healthy adult volunteers were collected in urine containers $(70 \mathrm{ml}$, Sarstedt AG \& Co., Nümbrecht, Germany; Urisafe, 3.0 liter polyethylene, VWR, Germany), filtered (VWR, syringe filter $25 \mathrm{~mm}, 0.2 \mu \mathrm{m}$ nylon membrane), aliquoted into CryoTubes ( $1.8 \mathrm{ml}$ and $4.5 \mathrm{ml}$, Nunc, Thermo Fisher Scientific Inc., Langenselbold, Germany) or UrineMonovettes ( $10 \mathrm{ml}$, Luer, Sarstedt AG \& Co.) and stored at $-20^{\circ} \mathrm{C}$. All participants had provided informed consent and the study protocol had been reviewed by the ethics committee of the Medical Faculty of the University of Munich. For sample preparation, an automated liquid handler (Gilson ASPEC GX-271, Middleton, Wisc., USA) was used. Each deep well (Riplate $1.2 \mathrm{ml}$ 96-deepwell, Ritter, Schwabmünchen, Germany) was prefilled with $460 \mu \mathrm{l}$ internal standard $\left(\mathrm{D}_{3}\right.$-creatinine $)$ in acetonitrile, then $40 \mu$ l of urine sample was added. The solution was mixed and incubated at $4^{\circ} \mathrm{C}$ for $60 \mathrm{~min}$ to complete protein precipitation. After centrifugation at $2,300 \mathrm{~g}$ for $10 \mathrm{~min}$ at $4^{\circ} \mathrm{C}$ (Rotina 38R, Hettich, Tuttlingen, Germany), $20 \mu \mathrm{l}$ of the supernatant were transferred into a second deep well plate (96 wells with $1.0 \mathrm{ml}$ each, polypropylene, Agilent, Germany) which was prefilled with $380 \mu$ l acetonitrile per well. The plates were sealed, shaken and placed in the autosampler of the LC-MS/MS system. 
Table 1. Instrument settings: MRM transitions and optimized ion path parameters of analyte and internal standard for the ABSciex API 2000

\begin{tabular}{lllllll}
\hline $\begin{array}{l}\text { Analyte/internal } \\
\text { standard }\end{array}$ & $\begin{array}{l}\text { Monoisotopic } \\
\text { mass, m/z }\end{array}$ & Q1, m/z & Q3, m/z & CEP, V & CE, V & CXP, V \\
\hline Creatinine_1 & 113.1 & 114.1 & 44.0 & 6 & 30 & 4 \\
Creatinine_2* & 113.1 & 114.1 & 86.0 & 8 & 61 & 2 \\
D3-creatinine_1 & 116.1 & 117.1 & 47.0 & 6 & 27 & 4 \\
D $_{3}$-creatinine_2* & 116.1 & 117.1 & 89.0 & 8 & 33 & 2 \\
\hline
\end{tabular}

Qualifier ions are marked with an asterisk $\left(^{*}\right)$; precursor ions (Q1) are selected in positive ion mode as $[\mathrm{M}+1]^{+}$; $\mathrm{Q} 3$ = product ion; $\mathrm{CEP}=$ collision cell entrance potential; $\mathrm{CE}=$ collision energy; $\mathrm{CXP}=$ collision cell exit potential.

\section{Instrumentation}

A triple quadrupole mass spectrometer ('API 2000', AppliedBiosystems/Sciex, Darmstadt, Germany) equipped with an electrospray ionization source ('TurboIonSpray') was used. FIA-MS/ MS was performed using an autosampler and pump of an HPLC system (Agilent Technologies, 1100 series HPLC system, Waldbronn, Germany). Data were acquired and processed using Analyst 1.5.1 software (Applied Biosystems/MDS, Toronto, Ont., Canada).

The electrospray source was operated in positive ion mode; optimized source parameters were: curtain gas 50 psi, collision gas 6 psi, ion spray voltage $5,000 \mathrm{~V}$, temperature $450^{\circ} \mathrm{C}$, nebulizer gas 40 psi, desolvation gas 80 psi. Compound-dependent ion path parameters were set to: declustering potential $20 \mathrm{~V}$, focusing potential $370 \mathrm{~V}$, entrance potential $10 \mathrm{~V}$, dwell time $100 \mathrm{~ms}$ and interface heater ON. Multiple reaction monitoring (MRM) transition parameters for the $[\mathrm{M}+1]^{+}$ions of analyte and internal standard, respectively, were optimized in positive ion mode. Quantifier and qualifier MRM transitions and ion path parameters for creatinine and $\mathrm{D}_{3}$-creatinine are summarized in table 1 .

Flow injection analysis was performed with a mixture of acetonitrile and water (50:50) acidified with $0.1 \%$ formic acid at an isocratic flow rate of $400 \mu \mathrm{l} / \mathrm{min}$. Total analysis time per sample was $0.4 \mathrm{~min}$ plus $0.6 \mathrm{~min}$ autosampler operation time.

\section{Validation Procedures}

In order to ensure selectivity and to exclude coelution bias, qualifier ion monitoring was implemented for both analyte and internal standard. Two different MRM transitions as alternative fragmentation routes were monitored for each precursor ion $[30$, 32]. The qualifier ion ratio (QIR) was calculated as the peak area ratio of these two MRM transitions (table 1) using Analyst 1.5.1 software. Reference QIR values were determined from pure standard solutions and compared to QIR of more than 270 native urine samples.

For accuracy research, urine samples collected from 5 healthy volunteers and one synthetic urine sample were divided into twelve aliquots each. Three of these aliquots were used unspiked and three were spiked with low $(0.75 \mathrm{mmol} / \mathrm{l})$, medium $(5 \mathrm{mmol} / \mathrm{l})$ or high concentration $(12 \mathrm{mmol} / \mathrm{l})$ of creatinine, respectively.

Injection repeatability was assessed using a series of eight urine preparations which were injected four times each. For test- ing intraday precision, six urine samples were prepared three times each in one batch. Interday precision was assessed by repeated preparation and analysis of three urine samples in two separate batches.

Pure creatinine standard dilutions in aqueous urea solution ( $255 \mathrm{mmol} / \mathrm{l})$ were used for external calibration of the isotope dilution assay (10 points within $0.25-40 \mathrm{mmol} / \mathrm{l})$. The calibration function was fitted by linear regression without weighting and the coefficient of determination $\left(\mathrm{R}^{2}\right)$ was calculated. Statistical analyses were performed with Statistica Version 10 (StatSoft GmbH, Hamburg, Germany).

As an external comparison method, creatinine was assessed by the Institute of Clinical Chemistry at the University of Munich Medical Center using the kinetic Jaffé method on a Cobas system (Roche Diagnostics, Mannheim, Germany), which is routinely applied in clinical chemistry.

\section{Results}

\section{Selectivity}

For verification of selectivity, reference QIR values were determined from pure standard solutions. For ease of comparison, relative QIR was calculated as the ratio of observed QIR to its respective reference QIR. QIR values calculated for 270 native urine samples were all within the range of $96-104 \%$ of reference QIR (fig. 1).

\section{Accuracy}

Known amounts of creatinine in low, medium and high concentration were added in small volumes to one synthetic and to five native urine samples. Accuracy was determined dividing the calculated concentrations of spiked samples by those of nonspiked samples; mean accuracy was $106.1 \pm 2.08 \%$ (synthetic urine: $108.0 \pm$ $2.72 \%$, mean of native urines: $105.8 \pm 2.09 \%$ ). Accuracy was constant over the entire range of concentrations ex- 


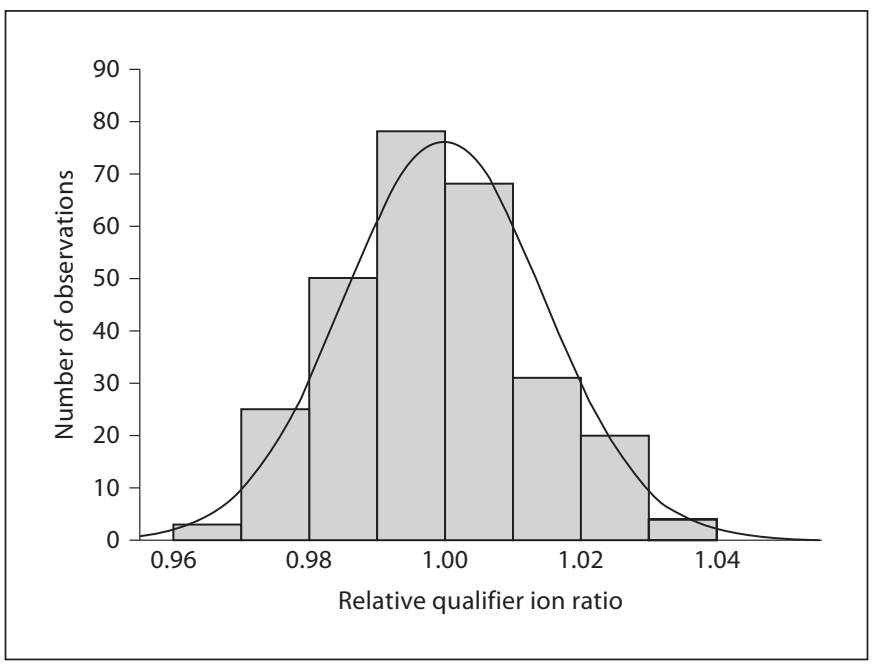

Fig. 1. QIR values calculated for 270 native urine samples were all within the range of $96-104 \%$ of reference QIR.

amined and exhibited no difference between native and synthetic urine matrix.

\section{Precision}

A series of four runs from eight urine samples was analyzed for injection repeatability yielding an average coefficient of variation of $1.0 \% \mathrm{CV}(0.7-1.6 \%)$. Intraday precision was evaluated on six urine samples of low, medium and high concentrations which were prepared three times in one batch; mean intraday precision was $1.2 \% \mathrm{CV}(0.5-$ $2.8 \%)$. Interday precision, determined from three urine samples of different concentration (high and low) analyzed in two batches, was $2.4 \%$ CV (1.7-3.4\%).

\section{Linearity}

The calibration function was fitted by linear regression without weighting $\left(y=0.0019+0.0001 x ; R^{2}=0.9988\right)$. The lowest point of the calibration curve $(0.25 \mathrm{mmol} / \mathrm{l})$ was defined as the limit of quantitation covering all concentrations determined from 270 spot and 24-hour urine samples, respectively.

\section{External Method Comparison}

Results obtained by the colorimetric assay were correlated with those of the FIA-MS/MS method ( $y=51.877$ $+1.1563 \mathrm{x} ; \mathrm{R}^{2}=0.9102$; fig. 2a). Concentration-dependent differences between the two assays were examined using a Bland-Altman plot (fig. 2b). All but 11 of 270 data points were lying within the confidence interval ( $\pm 1.96 \mathrm{SD})$.

\section{Discussion}

Determination of urinary creatinine by LC-MS/MS has already been described in the literature $[19,20]$. Park et al. [20] reported creatinine measurement in 24-hour urine samples after separation by reverse-phase liquid chromatography. Mass spectrometric analysis with and without purification on ion-exchange solid phase extraction (SPE) cartridges was applied by Huskova et al. [19]. In order to minimize analysis time and costs, we applied a radically simple approach using only two-step dilution of native urine in acetonitrile (1:250) for protein precipitation and dilution of sample matrix. FIA-MS/MS was then performed from the centrifuged supernatants without further preseparation by liquid chromatography or SPE. Extensive validation of our new method showed that the additional preparation procedures reported by others previously $[19,20]$ were unnecessary for reliable and accurate quantitation of urinary creatinine as we used FIAMS/MS combined with SIL-IS dilution and qualifier ion monitoring.

\section{Handling of Ion Suppression Effects}

Since creatinine is among the most abundant compounds in human urine, no dedicated sample preprocessing or separation prior to tandem mass spectrometry was deemed necessary. Creatinine can still be determined in heavily diluted urine samples where other matrix components are diluted so much that dedicated separation is not needed ('dilute and shoot'). Two-step isotope dilution in acetonitrile (1:250 in total) resulted both in protein precipitation and significant dilution of potentially interfering matrix components [33].

Ion suppression during electrospray ionization is inevitable under such conditions (as in most LC-MS/MS methods used in practice) [34], but dilution with adequately matched SIL-IS quantitatively corrects for these effects allowing accurate quantitation in the presence of significant ion suppression [28, 31, 33, 35]. Micová et al. [28] recently published findings that signal intensities in flow injection analysis were lower compared to liquid chromatography in consequence of ion suppression, but no influence on precision and accuracy of the assay was detectable due to the use of SIL-IS. $\mathrm{D}_{3}$-creatinine, which was used here as the SIL-IS, is very close to the ideal internal standard for creatinine as the mass difference of 3 $\mathrm{Da}$ is large enough to avoid MRM interferences of isotopologues but still small enough to retain nearly identical physicochemical properties. 


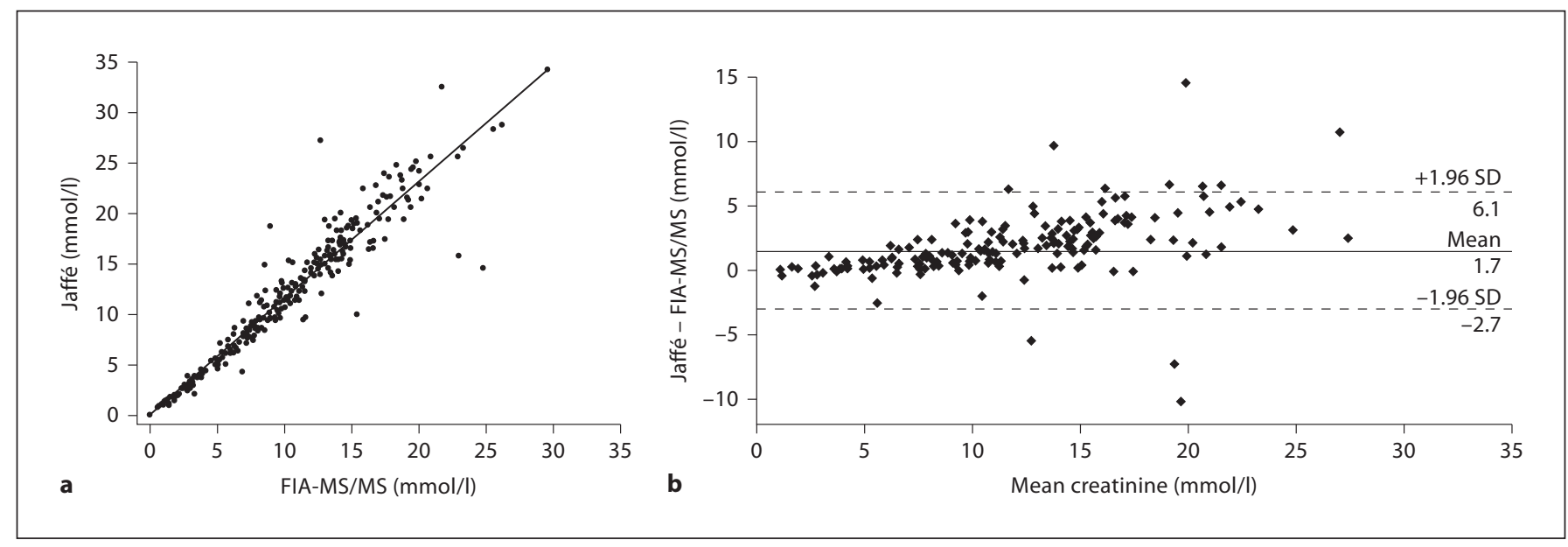

Fig. 2. a Results obtained by the colorimetric assay were correlated with those of the FIA-MS/MS method. b Concentration-dependent differences between the two assays were examined using a Bland-Altman plot. All but 11 of 270 data points were lying within the confidence interval ( $\pm 1.96 \mathrm{SD})$.

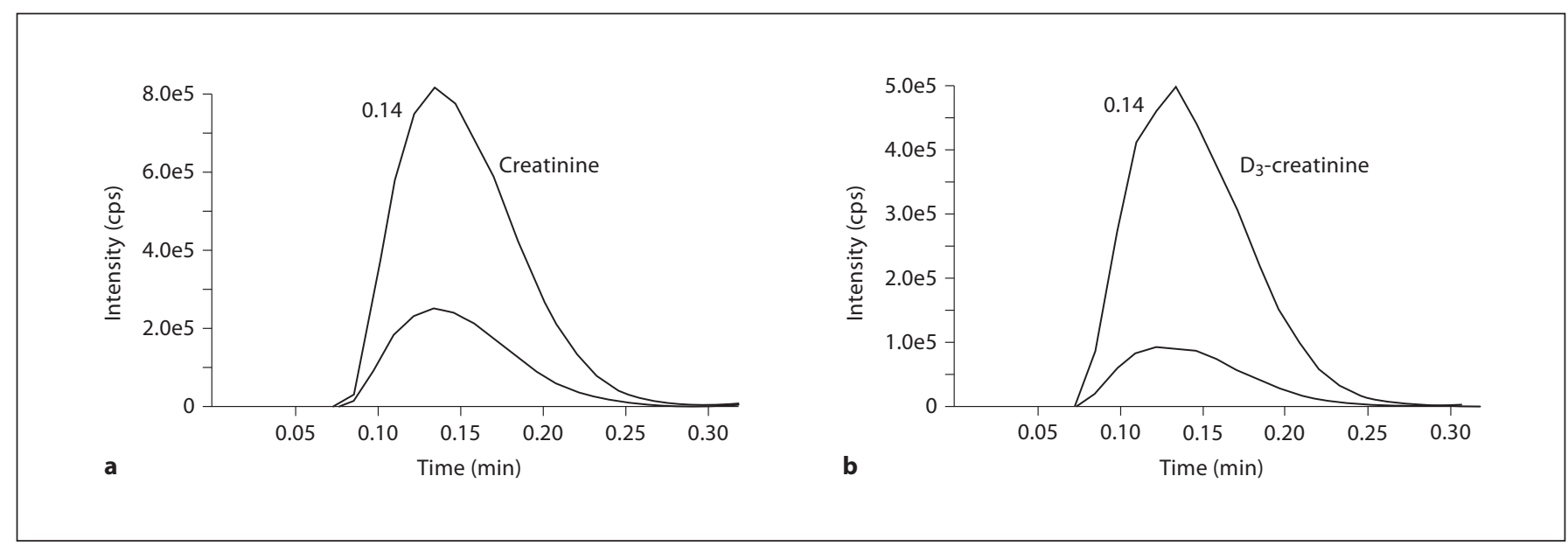

Fig. 3. Characteristic fingerprints of creatinine (a) and its internal standard $D_{3}$-creatinine (b), consisting of two specific MRM fragmentation routes each, were monitored in every sample. Both creatinine and its deuterated internal standard formed a flow injection peak at $0.14 \mathrm{~min}$.

\section{Selectivity}

Apart from ion suppression, LC-MS/MS assays may also be biased by coeluting compounds sharing similar mass transitions with the analyte of interest. Our approach of using fast flow injection analysis without chromatographic separation warranted special attention to possible coelution bias. We applied the concept of qualifier ion monitoring for ensuring unbiased quantitation of creatinine $[30,32]$. To that end, characteristic fingerprints of creatinine (fig. 3a) and its internal standard $\mathrm{D}_{3^{-}}$ creatinine (fig. 3b), consisting of two specific MRM fragmentation routes each, were monitored in every sample. Comparing QIR of native urine samples to those of pure standard solutions by calculating relative QIR showed no deviation (fig. 1), thus any coelution bias of the new methodology could be excluded. Unbiased quantitation by flow injection analysis was also presented by Johnson [35], who used a concept of comparing two pairs of analyte-to-IS mass transition ratios for confirmation of nonsusceptibility to interferences. Additionally, QIR could be 


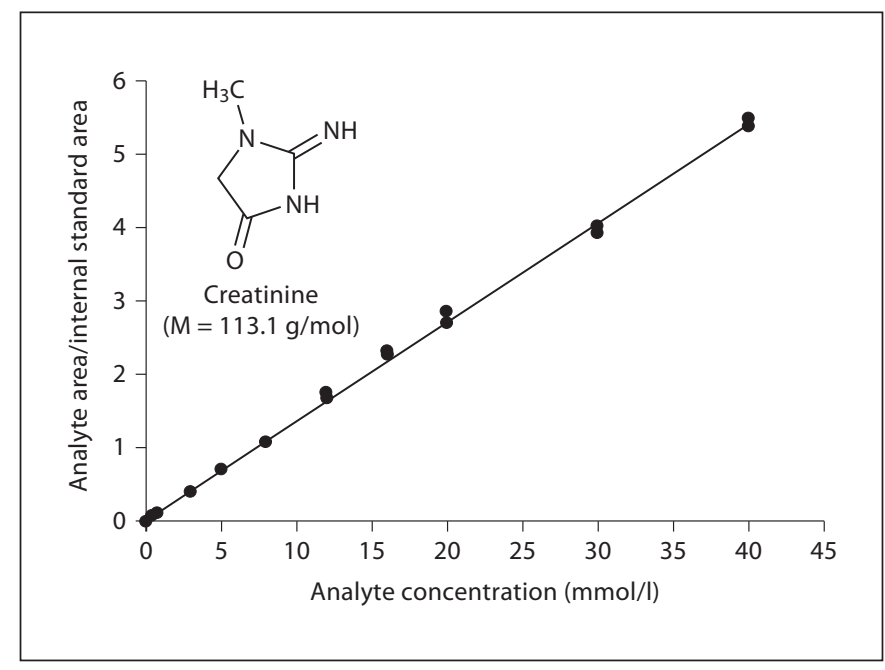

Fig. 4. Linear detector response over the whole calibration range from 0.25 to $40 \mathrm{mmol} / \mathrm{l}$, covering the full range of physiologically observed creatinine concentrations in human urine.

determined with high precision in standard and urine samples with average injection repeatability in native urine of $1.0 \% \mathrm{CV}$.

\section{Accuracy}

As no standard reference material was available, a standard addition procedure was performed for accuracy evaluation [36]. Low, medium and high concentrations of creatinine were spiked into synthetic and native urine for calculation of accuracy. Mean accuracy of $106 \%$ was found comparing spiked and nonspiked samples, which is comparable to previously published results [19]. Accuracy was constant over the entire concentration range and equal between native and synthetic matrix.

\section{Precision}

Results of precision research were compliant with widely accepted standard recommendations [36, 37], which demand precision determined at each concentration level not exceeding 15\% CV. Mean interday precision lower than $2.4 \% \mathrm{CV}$ was found for native urines, which is below results presented by Huskova et al. [19] applying SPE for sample preparation, and Park et al. [20] analyzing standard solution using LC-MS/MS. Results for mean intraday precision $(1.2 \% \mathrm{CV})$ and injection repeatability $(1.0 \% \mathrm{CV})$ analyzing native urine were even better than in previously published work $[19,20]$.

This FIA-MS/MS assay is based on a 'dilute and shoot' sample preparation, which is a radically simple protocol, but enables, if well optimized and validated, very reliable high-throughput mass spectrometric analysis [38]. In this case, the absence of SPE or liquid extraction and the omission of chromatographic separation does not hinder but in effect benefits superior precision of the assay due to elimination of possible sources of error and variability [26].

\section{Linearity}

Since creatinine-free urine samples were not available, we prepared creatinine dilution series in aqueous urea solution for external calibration. The dilution procedure was optimized to yield linear detector response over the whole calibration range from 0.25 to $40 \mathrm{mmol} / \mathrm{l}$ (fig. 4), covering the full range of physiologically observed creatinine concentrations in human urine $[39,40]$. The lowest creatinine concentration measured in 270 urine samples was $0.8 \mathrm{mmol} / \mathrm{l}$, so the lowest point of the calibration curve of $0.25 \mathrm{mmol} / \mathrm{l}$ was deemed properly selected.

\section{External Method Comparison}

As an external comparison method, colorimetric creatinine determination (Jaffé) on a Cobas Roche modular analyzer was compared with our FIA-MS/MS method. 270 urine samples with concentrations ranging from 0.8 to $34 \mathrm{mmol} / \mathrm{l}$ were analyzed with both methods. Results obtained by the FIA-MS/MS method were highly correlated with those of the colorimetric assay (fig. 2a), although there was a small but consistent bias towards higher values determined by the colorimetric method due to the well-known lack of selectivity (reaction with 'noncreatinine chromogens') of the Jaffé method $[15,16]$. Concentration-dependent differences between the two assays were examined using a Bland-Altman plot (fig. $2 \mathrm{~b}$ ), where 259 of 270 data points were within the confidence interval. Concentrations determined by the Jaffé assay were on average $1.7 \mathrm{mmol} / \mathrm{l}$ higher and exhibited creatinine concentration-dependent increase of positive bias (fig. 2b).

\section{Automation and High Throughput}

Our straightforward sample preparation protocol applying only sample dilution, centrifugation and transfer of supernatants was easily implemented by automated liquid handling. Use of the robotic pipetting device favorably influenced both speed of sample throughput and volume handling precision.

The flow rate was optimized for robust operation at $400 \mu \mathrm{l} / \mathrm{min}$. This relatively high flow rate was chosen to prevent obstruction of the electrospray needle by deposition of urinary components which had been observed at lower flow rates commonly used for flow injection analy- 
sis $(20-100 \mu \mathrm{l} / \mathrm{min})[19,28]$. To assess potential carryover, serial measurements of more than 130 injections were performed with blank samples measured at the beginning and end of each batch. All blanks revealed the same low intensity of background signals (signal to noise ratio $<1)$. Both creatinine and its deuterated internal standard formed a flow injection peak at $0.14 \mathrm{~min}$ (fig. 3). Flow injection analysis peak width was still sufficient to record 16 scan events for each of the quantifier or qualifier MRM transitions. After the creatinine signal, the flow was sustained until $0.4 \mathrm{~min}$ for thoroughly flushing the system. Autosampler operation time including needle washing accounted for additional $0.6 \mathrm{~min}$. The resulting total assay time of $1 \mathrm{~min}$ per sample allowed for very high throughput of more than 1,400 samples a day. Despite relatively high costs for equipment acquisition, this kind of FIA-MS/MS protocol can be very cost-effective for high-throughput screening of large numbers of samples. In comparison to previously published mass spectrometry methods $[19,20]$, this protocol has much lower costs per sample since expensive consumables as chromatography columns or SPE equipment are not used.

\section{Conclusions}

A rapid and simple FIA-MS/MS method for accurate quantitation of creatinine in human urine was developed. Isotope dilution tandem mass spectrometry with qualifier ion monitoring ensured unbiased quantitation and qualification of creatinine in every single sample. Precision and accuracy were in accordance with widely accepted bioanalytical standard recommendations and mostly superior to previously published protocols. Comparison with results measured by the colorimetric Jaffé assay routinely applied in clinical chemistry showed high agreement. Being characterized by a wide linear calibration range and excellent accuracy, the assay is applicable for the full range of creatinine concentrations observed in human urine. The method offers the advantages of low required sample volume as well as straightforward and fast sample preparation. Feasible throughput of more than 1,400 injections per day at low costs per sample makes it an attractive choice for creatinine determinations in large-scale epidemiological or clinical studies.

Although introduced more than 20 years ago, the full potential of FIA-MS/MS has so far not been fully recognized in analytical and clinical chemistry. Due to the omission of chromatographic preseparation, the occurrence of possibly interfering coelutions has to be care- fully assessed and excluded during validation. The concept of qualifier ion monitoring applied here effectively ensures proper qualification and unbiased quantification in each sample. FIA-MS/MS used with properly matched SIL-IS allows for very accurate and precise quantitative analysis which is often superior to conventional LC-MS/ MS. Improved scan speed of current mass spectrometers enables parallel quantification of dozens of analytes in a single run. Added benefit of multianalyte FIA-MS/MS determinations is the exceptionally high accuracy of interanalyte ratios within a single run. The only prerequisite for such multianalyte methods is to ensure that the expected concentration range of all analytes included fits into the dynamic range of the mass spectrometry system. Taken together, FIA-MS/MS is a very powerful tool for accurate quantification of biomolecules in complex matrices, which has the potential to revolutionize many applications of quantitative bioanalytical mass spectrometry including for example determination of pharmaceuticals, toxic chemicals or diagnostic biomarkers.

\section{Acknowledgements}

We express our sincere thanks to the Department of Clinical Chemistry, University of Munich Medical Center, for creatinine analysis by Jaffé method. This work was supported financially by the 'Kompetenznetz Adipositas' ('Competence Network for Adiposity') funded by the Federal Ministry of Education and Research (FKZ: 01GI1126). Further support by the Commission of the European Communities, within the 7th Framework Programme, NUTRIMENTHE, FP7-212652 and by the Munich Center of Health Sciences (McHealth) is gratefully acknowledged. This paper does not necessarily reflect the views of the Commission and in no way anticipates future policy in this area. B.K. is a recipient of the Freedom to Discover Award of the BristolMyers Squibb Foundation, New York, USA. The presented work is part of a PhD thesis accomplished by M.N. at the Medical Faculty of the Ludwig Maximilians University of Munich.

References Dalal M, Jardack P, Traber MG, Ferrucci L, Arab L: Dietary intake associated with serum versus urinary carboxymethyl-lysine, a major advanced glycation end product, in adults: the energetics study. Eur J Clin Nutr 2012;66:3-9.

2 Rossi MC, Nicolucci A, Pellegrini F, Comaschi M, Ceriello A, Cucinotta D, Giorda C, Pomili B, Valentini U, Vespasiani G, De CosmoS: Obesity and changes in urine albumin/ creatinine ratio in patients with type 2 diabetes: the demand study. Nutr Metab Cardiovasc Dis 2010;20:110-116. 
\$3 Lutz U, Bittner N, Lutz RW, Lutz WK: Metabolite profiling in human urine by LC-MS/ MS: method optimization and application for glucuronides from dextromethorphan metabolism. J Chromatogr B Analyt Technol Biomed Life Sci 2008;871:349-356.

$\checkmark 4$ Lee KF, Chung WY, Benzie IF: Urine 8-oxo7,8-dihydro-2'-deoxyguanosine (8-oxodG), a specific marker of oxidative stress, using direct, isocratic LC-MS/MS: method evaluation and application in study of biological variation in healthy adults. Clin Chim Acta 2010;411:416-422.

5 Bhat SH, Gelhaus SL, Mesaros C, Vachani A, Blair IA: A new liquid chromatography/mass spectrometry method for 4-(methyl nitrosamino)-1-(3-pyridyl)-1-butanol (NNAL) in urine. Rapid Commun Mass Spectrom 2011; 25:115-121.

6 Wyss M, Kaddurah-Daouk R: Creatine and creatinine metabolism. Physiol Rev 2000;80: 1107-1213.

-7 Tsikas D, Wolf A, Mitschke A, Gutzki FM, Will W, Bader M: GC-MS determination of creatinine in human biological fluids as pentafluorobenzyl derivative in clinical studies and biomonitoring: inter-laboratory comparison in urine with Jaffé, HPLC and enzymatic assays. J Chromatogr B Analyt Technol Biomed Life Sci 2010;878:2582-2592.

$\checkmark 8$ Rehberg PB: Studies on kidney function: the rate of filtration and reabsorption in the human kidney. Biochem J 1926;20:447-460.

-9 Liotta E, Gottardo R, Bonizzato L, Pascali JP, Bertaso A, Tagliaro F: Rapid and direct determination of creatinine in urine using capillary zone electrophoresis. Clin Chim Acta 2009;409:52-55.

$\checkmark 10$ Narayanan S, Appleton HD: Creatinine: a review. Clin Chem 1980;26:1119-1126.

$\checkmark 11$ Guder WG, Hoffmann GE, Hubbuch A, Poppe WA, Siedel J, Price CP: Multicentre evaluation of an enzymatic method for creatinine determination using a sensitive colour reagent. J Clin Chem Clin Biochem 1986; 24:889-902.

12 Sugita O, Uchiyama K, Yamada T, Sato T, Okada M, Takeuchi K: Reference values of serum and urine creatinine, and of creatinine clearance by a new enzymatic method. Ann Clin Biochem 1992;29:523-528.

13 Linnet K, Bruunshuus I: HPLC with enzymatic detection as a candidate reference method for serum creatinine. Clin Chem 1991;37:1669-1675.

14 Jaffé M: Ueber den Niederschlag, welchen Pikrinsäure im normalen Harn erzeugt, und über eine neue Reaction des Kreatinins. Zeitschrift für Physiologische Chemie 1886;10: 391-400.

15 Weber JA, van Zanten AP: Interferences in current methods for measurements of creatinine. Clin Chem 1991;37:695-700.

$\checkmark 16$ Hussain I, Tariq MI, Siddiqui HL: Structure elucidation of chromogen resulting from Jaffé's reaction. J Chem Soc Pak 2009;31:937948.
17 Burke DG, MacLean PG, Walker RA, Dewar PJ, Smith-Palmer T: Analysis of creatine and creatinine in urine by capillary electrophoresis. J Chromatogr B Biomed Sci Appl 1999; 732:479-485.

18 Jen JF, Hsiao SL, Liu KH: Simultaneous determination of uric acid and creatinine in urine by an eco-friendly solvent-free high performance liquid chromatographic method. Talanta 2002;58:711-717.

19 Huskova R, Chrastina P, Adam T, Schneiderka P: Determination of creatinine in urine by tandem mass spectrometry. Clin Chim Acta 2004;350:99-106.

20 Park EK, Watanabe T, Gee SJ, Schenker MB, Hammock BD: Creatinine measurements in $24 \mathrm{~h}$ urine by liquid chromatography - tandem mass spectrometry. J Agric Food Chem 2008;56:333-336.

21 Chace DH, Millington DS, Terada N, Kahler SG, Roe CR, Hofman LF: Rapid diagnosis of phenylketonuria by quantitative analysis for phenylalanine and tyrosine in neonatal blood spots by tandem mass spectrometry. Clin Chem 1993;39:66-71.

22 Millington DS, Kodo N, Norwood DL, Roe CR: Tandem mass spectrometry: a new method for acylcarnitine profiling with potential for neonatal screening for inborn errors of metabolism. J Inherit Metab Dis 1990; 13:321-324.

23 Chace DH, Kalas TA: A biochemical perspective on the use of tandem mass spectrometry for newborn screening and clinical testing. Clin Biochem 2005;38:296-309.

24 McHugh DM, Cameron CA, Abdenur JE, et al: Clinical validation of cutoff target ranges in newborn screening of metabolic disorders by tandem mass spectrometry: a worldwide collaborative project. Genet Med 2011;13: 230-254.

25 John H, Eddleston M, Clutton RE, Worek F, Thiermann H: Simultaneous quantification of the organophosphorus pesticides dimethoate and omethoate in porcine plasma and urine by LC-ESI-MS/MS and flow-injectionESI-MS/MS. J Chromatogr B Analyt Technol Biomed Life Sci 2010;878:1234-1245.

26 Nanita SC, Pentz AM, Bramble FQ: Highthroughput pesticide residue quantitative analysis achieved by tandem mass spectrometry with automated flow injection. Analytical Chemistry 2009;81:3134-3142.

27 Nanita SC, Stry JJ, Pentz AM, McClory JP, May JH: Fast extraction and dilution flow injection mass spectrometry method for quantitative chemical residue screening in food. J Agric Food Chem 2011;59:7557-7568.

28 Micová K, Friedecky D, Faber E, Polynkova A, Adam T: Flow injection analysis vs. ultra high performance liquid chromatography coupled with tandem mass spectrometry for determination of imatinib in human plasma. Clin Chim Acta 2010;411:1957-1962.
29 Song F, El-Demerdash A, Lee S-JSH, Smith RE: Fast screening of lovastatin in red yeast rice products by flow injection tandem mass spectrometry. J Pharm Biomed Anal 2012;57: 76-81.

30 Delatour T, Mottier P, Gremaud E: Limits of suspicion, recognition and confirmation as concepts that account for the confirmation transitions at the detection limit for quantification by liquid chromatography-tandem mass spectrometry. J Chromatogr A 2007; 1169:103-110.

31 Matuszewski BK: Standard line slopes as a measure of a relative matrix effect in quantitative HPLC-MS bioanalysis. J Chromatogr B Analyt Technol Biomed Life Sci 2006;830: 293-300.

32 Harder U, Koletzko B, Peissner W: Quantification of 22 plasma amino acids combining derivatization and ion-pair LC-MS/MS. J Chromatogr B Analyt Technol Biomed Life Sci 2011;879:495-504.

- 33 Van Eeckhaut A, Lanckmans K, Sarre S, Smolders I, Michotte Y: Validation of bioanalytical LC-MS/MS assays: evaluation of matrix effects. J Chromatogr B Analyt Technol Biomed Life Sci 2009;877:2198-2207.

34 Taylor PJ: Matrix effects: the Achilles heel of quantitative high-performance liquid chromatography-electrospray-tandem mass spectrometry. Clin Biochem 2005;38:328334.

35 Johnson DW: An acid hydrolysis method for quantification of plasma free and total carnitine by flow injection tandem mass spectrometry. Clin Biochem 2010;43:1362-1367.

36 US Department of Health and Human Services, Food and Drug Administration (FDA), Center for Drug Evaluation and Research (CDER), Center for Veterinary Medicine (CVM): Guidance for industry: bioanalytical method validation. BP, 2001.

37 US Department of Health and Human Services, Food and Drug Administration (FDA), Center for Drug Evaluation and Research (CDER), Center for Biologics Evaluation and Research (CBER): Guidance for industry: Q2B validation of analytical procedures: methodology. International Conference on Harmonisation (ICH) of Technical Requirements for Registration of Pharmaceuticals for Human Use, 1996.

-38 Nanita SC: High-throughput chemical residue analysis by fast extraction and dilution flow injection mass spectrometry. Analyst 2011;136:285-287.

- 39 Barr DB, Wilder LC, Caudill SP, Gonzalez AJ, Needham LL, Pirkle JL: Urinary creatinine concentrations in the U.S. population: implications for urinary biologic monitoring measurements. Environ Health Perspect 2005;113:192-200.

40 Arndt T: Urine-creatinine concentration as a marker of urine dilution: reflections using a cohort of 45,000 samples. Forensic Sci Int 2009; 186:48-51. 